University of Nebraska - Lincoln

DigitalCommons@University of Nebraska - Lincoln

Faculty Publications: Materials Research

Science and Engineering Center

Materials Research Science and Engineering

Center

2006

\title{
Fast and Slow Magnetization Processes in Magnetic Recording Media
}

Jian Zhou

University of Nebraska-Lincoln, jzhou@unlserve.unl.edu

Ralph Skomski

University of Nebraska at Lincoln, rskomski2@unl.edu

Steven A. Michalski

University of Nebraska - Lincoln, smichalski2@unl.edu

Roger D. Kirby

University of Nebraska-Lincoln, rkirby1@unl.edu

David J. Sellmyer

University of Nebraska-Lincoln, dsellmyer@unl.edu

Follow this and additional works at: https://digitalcommons.unl.edu/mrsecfacpubs

Part of the Materials Science and Engineering Commons

Zhou, Jian; Skomski, Ralph; Michalski, Steven A.; Kirby, Roger D.; and Sellmyer, David J., "Fast and Slow Magnetization Processes in Magnetic Recording Media" (2006). Faculty Publications: Materials Research Science and Engineering Center. 76.

https://digitalcommons.unl.edu/mrsecfacpubs/76

This Article is brought to you for free and open access by the Materials Research Science and Engineering Center at DigitalCommons@University of Nebraska - Lincoln. It has been accepted for inclusion in Faculty Publications: Materials Research Science and Engineering Center by an authorized administrator of DigitalCommons@University of Nebraska - Lincoln. 


\title{
FAST AND SLOW MAGNETIZATION PROCESSES IN MAGNETIC RECORDING MEDIA
}

\author{
J. Zhou, R. Skomski, S. Michalski, R. D. Kirby, and D. J. Sellmyer \\ Department of Physics and Astronomy and Center for Materials Research and Analysis, \\ University of Nebraska, Lincoln, NE 68588
}

\begin{abstract}
Information loss due to thermal activation is a major concern in ultrahigh-density magnetic recording media. The usually considered mechanism is thermally activated magnetization reversal over micromagnetic energy barriers. However, micromagnetic approaches ignore local anisotropy fluctuations, which translate into a time-dependent reduction of the remanent magnetization. The effect is negligibly small in macroscopic magnets but becomes important on a scale of a few nanometers.
\end{abstract}

\section{INTRODUCTION}

Magnetic recording has been a driving force in nanotechnology and electronics. The key advantage is the high storage density, corresponding to bit sizes much smaller than the wavelength of visible light. However, a fundamental bit-size limit is given by the thermal stability of the stored information [1]. In very small grains or particles, thermal activation leads to local magnetization reversal and to the decay of the stored information. In macroscopic magnets, there is a clear separation of time scales between fast atomic or intrinsic processes and slow extrinsic magnetization processes. Intrinsic properties can be treated by equilibrium statistical mechanics. For example, the local magnetic anisotropy $K_{1}(\mathbf{r})$ can be replaced by the time or ensemble average $\left\langle K_{1}(\mathbf{r})\right\rangle$ $[1,2]$. By contrast, extrinsic properties are related to hysteresis and often far from equilibrium $[3,4]$.

The question arises how magnetic systems behave on a length scale of a very few nanometers, where intrinsic phenomena become important. This applies, for example, to single-phase particles, core-shell structures, and exchange-coupled hard-soft structures, which have attracted renewed attention $[5,6]$ as magnetic-recording materials. This paper starts with a brief analysis of magnetization modes in the structures and then outlines how fluctuations of intrinsic properties affect the time-dependence magnetic properties.

\section{NUCLEATION MODES IN COMPOSITE NANOPARTICLES}

The simplest model of magnetization reversal, the Stoner-Wohlfarth model, describes uniformly magnetized small particles of volume $V$. In zero field, it yields the energy barrier $\left\langle K_{1}>V\right.$ and a dynamics described by the Arrhenius law $\exp \left(-<K_{1}>V / k_{\mathrm{B}} T\right)$. In two-phase particles (Fig. 1), magnetization inhomogenities are essential, because the soft phase switches earlier than the hard phase. Figures 1(b) shows the nucleation mode, that is, the magnetization deviation from $\boldsymbol{M}=M_{\mathrm{s}} \boldsymbol{e}_{\mathrm{z}}$ at the onset of magnetization reversal. 


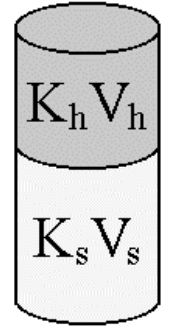

(a)
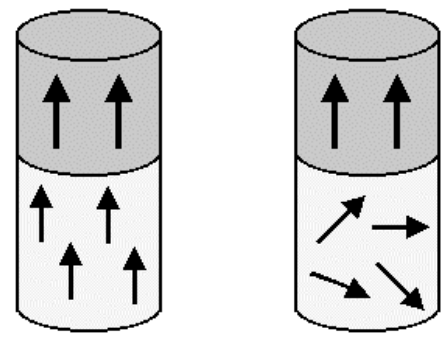

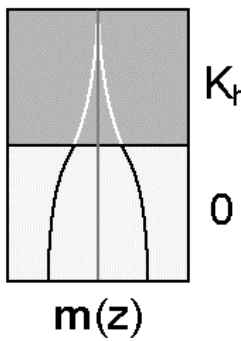

(b)

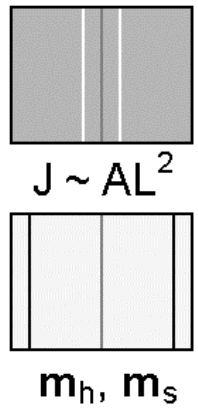

(c)

Fig. 1. Two-phase nanoparticle consisting of hard (h) and soft (s) phases: (a) structure, (b) magnetization modes, and (c) two-particle model. In (b), a magnetization tail reaches into the hard phase and reduces the energy barrier of the particle.

The model of Fig. 1(c) approximates the composite nanoparticle by two particles interacting via an effective exchange $J \sim 1 / L^{2}$, where $\mathrm{L}$ is the particle size. The model has been used to discuss the coercivity of small permanent-magnet particles [7], but it can also be used to estimate the spin-wave dynamics of the system, in analogy to the wellknown treatment of ferromagnetic resonance by Kittel $[8,9]$. The solution of the problem amounts to the diagonalization of a $2 \times 2$ matrix whose nondiagonal matrix elements are proportional to $J$ and where the demagnetization field of [8] is replaced by a more general anisotropy field. Figure 2 shows the predicted resonance frequencies. For large particles, $J \sim 1 / L^{2}$ is negligible, and the system behaves like a superposition of hard and soft phases. On decreasing particle size, the modes hybridize and change their character. The lowest-lying mode can now be considered ferromagnetic, as in Fig. 1(b-c), whereas the excited mode is 'antiferromagnetic' with an oscillation $180^{\circ}$ out of phase.

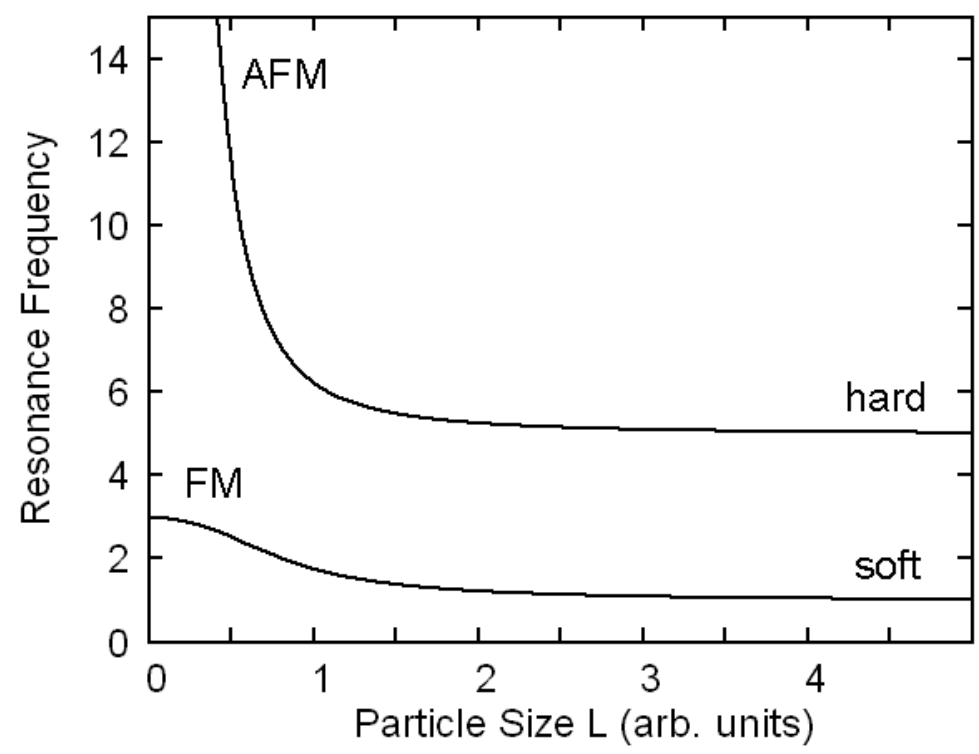

Fig. 2. Resonance modes in two-phase nanoparticles. The calculation is based on the model of Fig. 1(c). 


\section{ROLE OF FLUCTUATIONS}

Micromagnetic approaches, such as Stoner-Wohlfarth model and the models of Fig. 1 , start from thermally averaged intrinsic properties such as $\left\langle\mathrm{K}_{1}(\mathbf{r})\right\rangle$. This is a good approximation for macroscopic magnets but not necessarily for small nanoparticles. Figure 3 illustrates this point for a specific class of systems, namely rare-earth transitionmetal intermetallics, for example $\mathrm{SmCo}_{5}$ and $\mathrm{Sm}_{2} \mathrm{Fe}_{17} \mathrm{~N}_{3}$. In the intermetallics, the anisotropy is provided by the rare-earth sublattice, which consists of $\mathrm{Sm}^{3+}$ ions. It is wellknown [10] that the finite-temperature average anisotropy $\left\langle K_{1}(T)>\sim\left(3<J_{\mathrm{z}}^{2}\right\rangle-J^{2}-J\right)$ is caused by intramultiplet excitations $\left(J_{z}<J\right)$ of the type shown in Fig. 3(b). On a micromagnetic level, this corresponds to the Arrhenius law $\tau=\tau_{0} \exp \left(-\mathrm{E}_{\mathrm{a}}\left(<K_{1}(T)>k_{\mathrm{B}} T\right)\right)$

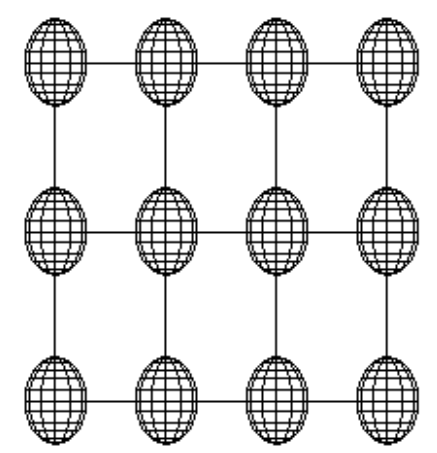

(a)

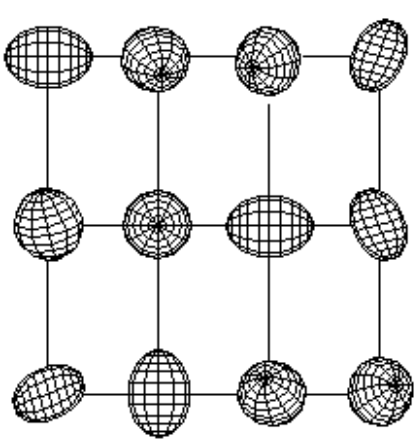

(b)

Fig. 3. Spin structure of rare-earth sublattices at (a) zero and (b) finite temperatures. The ellipsoids correspond to the $4 \mathrm{f}$ charge distribution of the $\mathrm{Sm}^{3+}$ ions, and the rare-earth moments are parallel to the axes of revolution.

However, nonzero fluctuations $\left\langle\left(K_{1}(\mathbf{r})-\left\langle K_{1}>\right)^{2}\right\rangle\right.$ facilitate the magnetization reversal by reducing the energy barriers, rather than driving the magnetization over the energy barrier. Figure 4 illustrates that the size of the maximum or 'giant' fluctuations increases with waiting time. In macroscopic systems, these fluctuations can be ignored, but in very small particles, they affect the long-time behavior of the magnet and add to the magnetization decay predicted from $<K_{1}>$. The effective anisotropy $K_{\text {eff }}=\left\langle K_{1}\right\rangle-\delta K$ obeys
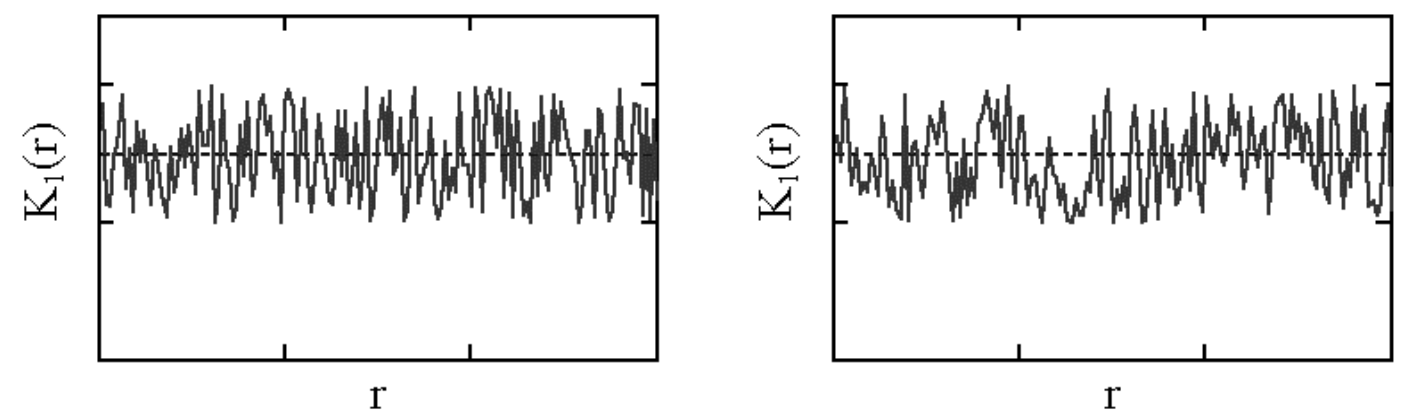

Fig. 4. Anisotropy fluctuations. Giant fluctuations, such as the negative fluctuation near the center of the right figure, require long waiting times. 


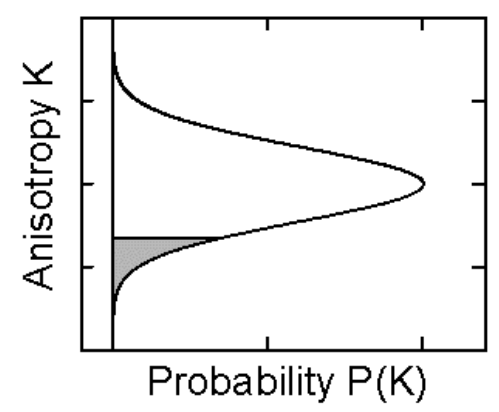

Fig. 5. Gaussian anisotropy distribution, averaged over a volume $\xi^{d}$. The gray area is inversely proportional to the waiting time.

$$
K_{\text {eff }}(T, \mathrm{t})=<K_{1}>-\delta K_{\mathrm{o}} \sqrt{2 \ln \left(\tau / \tau^{*}\right)}
$$

where $\delta K_{\mathrm{o}}$ and $\tau^{*}$ depend on temperature and one the volume over $\xi^{\mathrm{d}}$ over which the anisotropy is averaged. As it will be explained elsewhere, Eq. (1) involves a complicated random-anisotropy and time averaging over all spin configurations. In a simple interpretation, Fig. 5, the logarithmic time dependence of $K_{\text {eff }}$ reflects the low-lying parts of the Gaussian anisotropy distribution, which is a consequence of the mechanism of Fig. 3(b).

To quantify the effect in an approximate manner, we have performed a numerical simulation using the OOMMF code by NIST (http://math.nist.gov/oommf). Thermal excitations enter the calculation in form of a random anisotropy whose magnitude depends on the waiting time. In other words, we temporarily 'freeze' the dynamic spin configuration to check whether the thermal disorder is sufficient to realize magnetization reversal in a given reverse field. The investigated cylindrical single-phase particle has a diameter of $8 \mathrm{~nm}$ and a height of $10 \mathrm{~nm}$. The used materials parameters are $\left\langle K_{1}\right\rangle=5$ $\mathrm{MJ} / \mathrm{m}^{3}, A=10 \mathrm{pJ} / \mathrm{m}$, and $M_{\mathrm{s}}=1100 \mathrm{MA} / \mathrm{m}$. In the simulations $\delta K=1-3 \mathrm{MJ} / \mathrm{m}^{3}$. Figure 6 shows the hysteresis of the particle for different waiting times (sweep rates).

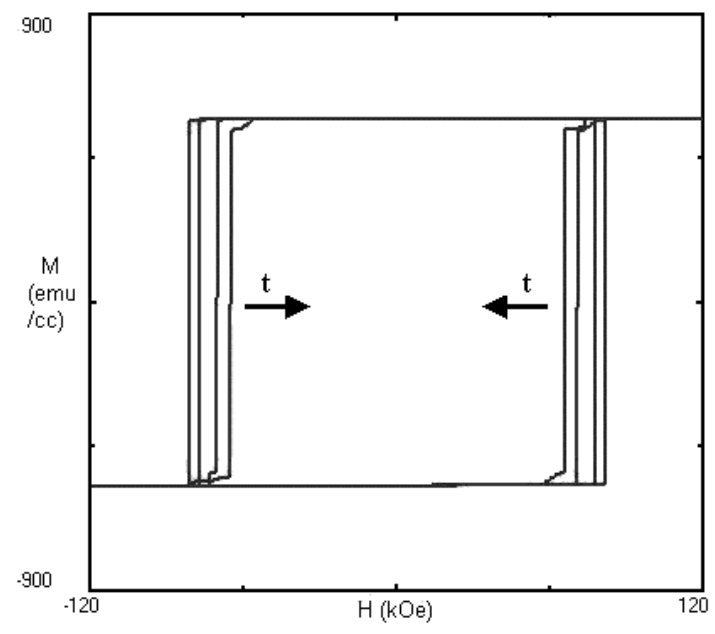

Fig. 6. Time-dependence of the hysteresis loop in the 'frozen' or 'snapshot' approximation. 


\section{DISCUSSION AND CONCLUSIONS}

It is instructive to compare the present local-anisotropy picture to the StonerWohlfarth reversal in small particles. In the Stoner-Wohlfarth picture, Fig. 7(a), the spins remain parallel during magnetization reversal, but spin disorder (b) opens another channel for reversal. Usually, the random contribution is small, but in particles smaller than $10 \mathrm{~nm}$, it may become nonnegligible. For example, in thins wires of cross section $\pi R^{2}$, the activation volume scales as $R^{2}$ and approaches zero for very small $R$. In this limit, local randomness becomes the main consideration.

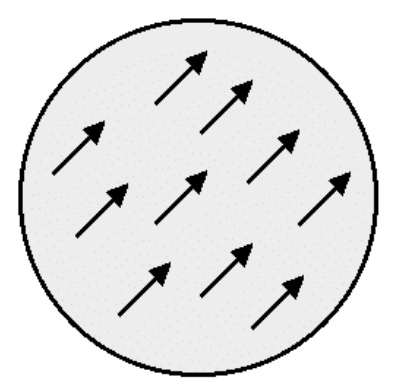

(a)

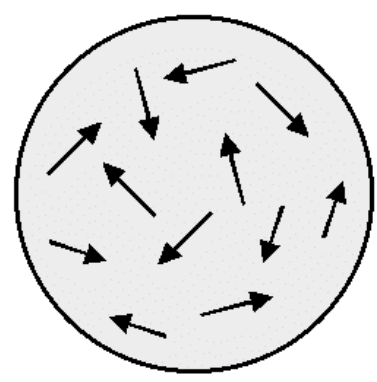

(b)

Fig. 7. Comparison of Stoner-Wohlfarth model (a) and atomically randomized spins (b).

Naturally, our quasistatic or 'frozen' Gaussian approximation is relatively crude. It can be shown that Eq. (1) requires some time averaging to ensure that the giant anisotropy fluctuation exists for about $1 \mathrm{~ns}$, so that thermal reversal can occur. In Eq. (1), this averaging appears in for of the time constant $\tau^{*}$, which is larger than the time scale of atomic anisotropy fluctuations and reduces $\delta K$. This procedure ignores, for example, fast

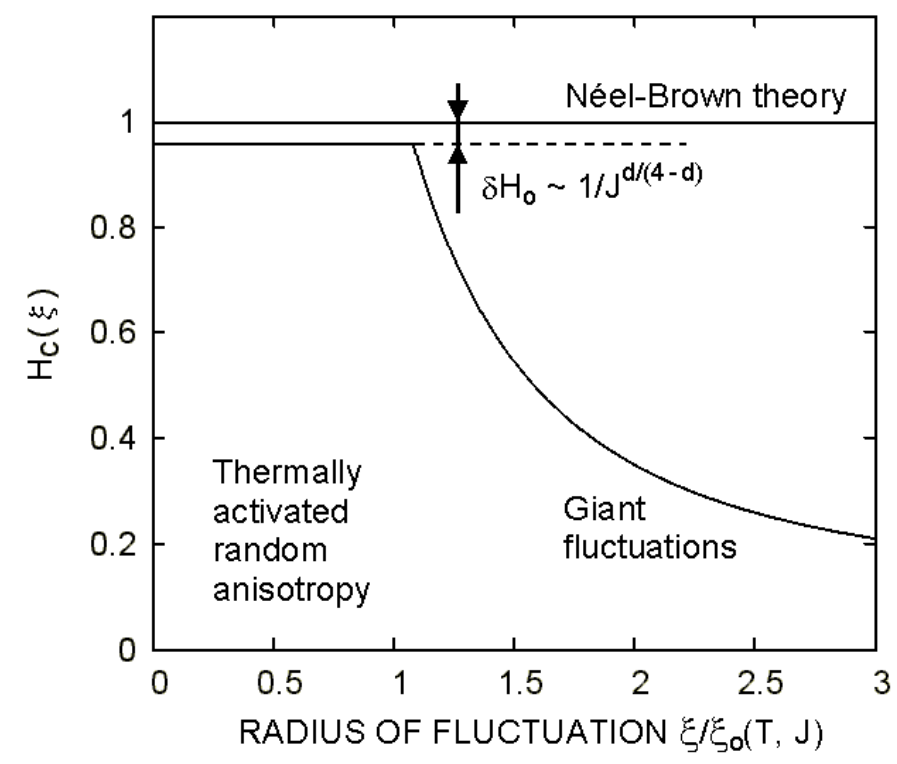

Fig. 8. Coercivity as a function of the size of bulk anisotropy fluctuations. 
magnetization processes that have the character of spin precessions. The corresponding fast magnetization dynamics in nanostructures and multilayers is currently under experimental and theoretical investigation, but its discussion goes beyond the scope of this paper.

To gauge the effect of the anisotropy fluctuations, it is also necessary to consider their size $\xi$. Atomic-scale spin disorder occurs frequently but has very little effect on the anisotropy. To have a significant effect on the magnetization reversal, the fluctuation must comprise many atoms. In the simulations leading to Fig. 6, this effect is automatically included. Analytically, it is necessary to nucleation field in a random potential, as outlined in [3]. Figure 8 shows the corresponding result.

In conclusion, we have investigated how local anisotropy fluctuations affect the magnetization dynamics of small particles. Our preliminary numerical and analytic calculations quantify the effect by using the 'frozen' or 'snapshot' approximation, which maps the finite-temperature problem onto a problem onto a static but disordered system. The local fluctuations are most pronounced in small grains or particles and negatively affect the thermal stability. They amount to fluctuating energy barriers and reduce the remanent magnetization beyond the Arrhenius-Néel-Brown predictions. The effect, which we have treated in a static approximation, adds to the dynamics based on micromagnetic free energies, such as Arrhenius-Néel-Brown activation and ferromagnetic resonance.

\section{ACKNOWLEDGEMENT}

This research is supported by the W. M. Keck Foundation, NSF-MRSEC, INSIC, and CMRA.

\section{REFERENCES}

[1] D. Weller and A. Moser, IEEE Trans. Magn. 35, 4423-4439 (1999).

[2] D. J. Sellmyer and R. Skomski (Eds.), "Advanced Magnetic Nanostructures", Springer, Berlin 2005.

[3] R. Skomski, J. Phys.: Condens. Matter 15, R841 (2003).

[4] D. Givord and M. F. Rossignol, in: "Rare-Earth Iron Permanent Magnets", Ed.: J. M. D. Coey, University Press, Oxford 1996, p. 218-285.

[5] R. H. Victora and X. Shen, IEEE Trans. Magn. 41, 537 (2005).

[6] D. Suess, T. Schrefl, S. Fähler, M. Kirschner, G. Hrkac, F. Dorfbauer, and J. Fidler, Appl. Phys. Lett. 87, 012504 (2005).

[7] J. M. D. Coey and R. Skomski, Physica Scripta T49, 315 (1993).

[8] C. Kittel, "Introduction to Solid-State Physics", Wiley, New York 1986.

[9] M. I. Chipara, R. Skomski, and D. J. Sellmyer, J. Magn. Magn. Mater. 249, 246 (2002).

[10] R. Skomski and J. M. D. Coey, "Permanent Magnetism", Institute of Physics, Bristol 1999. 